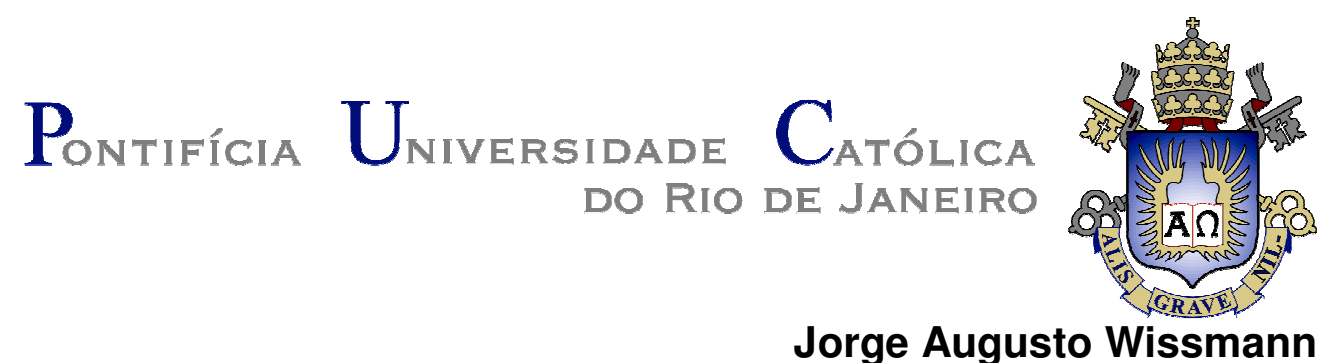

\title{
Estudo de Vigas de Aço com Aberturas de Alma Tipo Alveolar, Litzka e Vigas Expandidas em Altura
}

\section{Dissertação de Mestrado}

Dissertação apresentada como requisito parcial para obtenção do título de Mestre pelo Programa de PósGraduação em Engenharia Civil da PUC-Rio.

Orientador: Sebastião Arthur Lopes de Andrade 


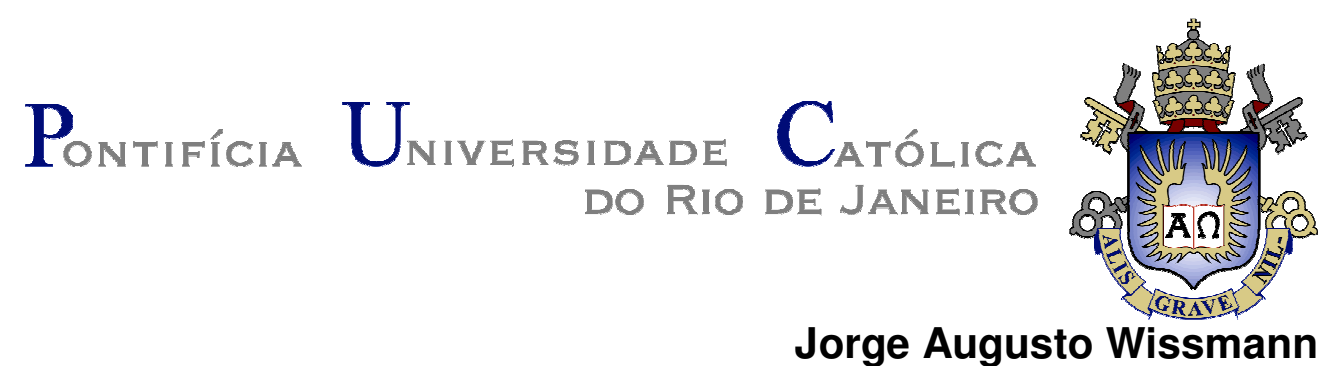

\title{
Estudo de Vigas de Aço com Aberturas de Alma Tipo Alveolar, Litzka e Vigas Expandidas em Altura
}

Dissertação apresentada como requisito parcial para obtenção do título de Mestre pelo Programa de PósGraduação em Engenharia Civil da PUC-Rio. Pendente de Aprovação pela Comissão Examinadora abaixo.

\author{
Sebastião Arthur Lopes de Andrade \\ Orientador \\ Departamento de Engenharia Civil - PUC-Rio \\ Pedro Colmar Gonçalves da Silva Vellasco \\ Departamento de Estruturas e Fundações - UERJ \\ José Guilherme Santos da Silva \\ Departamento de Engenharia Mecânica - UERJ \\ Raul Rosas e Silva \\ Departamento de Engenharia Civil - PUC-Rio \\ José Eugênio Leal \\ Coordenador Setorial do Centro Técnico Científico - PUC-Rio
}


Todos os direitos reservados. É proibida a reprodução total ou parcial do trabalho sem autorização da universidade, do autor e do orientador.

Ficha Catalográfica

Wissmann, Jorge Augusto

Estudo de vigas de aço com aberturas de alma tipo alveolar, Litzka e vigas expandidas em altura / Jorge Augusto Wissmann ; orientador: Sebastião Arthur Lopes de Andrade. - 2009.

139 f. : il. (color.) ; $30 \mathrm{~cm}$

Dissertação (Mestrado em Engenharia Civil)-Pontifícia Universidade Católica do Rio de Janeiro, Rio de Janeiro, 2009.

Inclui bibliografia

1. Engenharia civil - Teses. 2. Análise pelo método dos elementos finitos. 3. Mecanismo de Vierendeel. 4. Abertura na alma. 5. Curvas de interação flexão-cisalhamento. 6. Não linearidade física e geométrica. 7. Vigas de aço. 8. Vigas expandidas. 9. Análise paramétrica. I. Andrade, Sebastião A. L. de. II. Pontifícia Universidade Católica do Rio de Janeiro. Departamento de Engenharia Civil. III. Título.

CDD: 624 


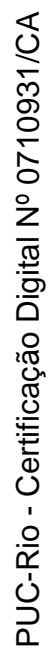

Aos meus pais e toda minha família, pelo amor e apoio. 


\section{Agradecimentos}

Aos meus pais e minha família, pelo apoio a minha escolha em dedicar mais alguns anos aos estudos, sem poderem contar com meu apoio próximo em uma época de problemas.

A Édina Michelli Allebrandt, que esteve ao meu lado durante esse período, apoiando e escutando nos momentos de necessidade.

Aos professores Fábio Luiz Willrich e Humberto Correia Lima Júnior pelo apoio e recomendação ao início do mestrado.

A Dilnei Schmidt, colega e amigo, pela dedicação de seu tempo ao me ensinar a como utilizar o software de elementos finitos. Sem sua ajuda a pesquisa teria sido comprometida.

A Arthur Medeiros, João Soto Krause e Paul Antezana que durante toda a convivência desta época foram mais que colegas, se tornaram amigos também.

A Sebastião Arthur Lopes de Andrade, orientador da presente dissertação, que protagonizou seu papel direcionando leituras e autores, guiando pensamentos e idéias, assim como auxiliando em momentos de necessidade intelectual durante nossos encontros.

As agências de fomento CNPq e CAPES, que propiciaram condições financeiras, sem as quais a pesquisa e a obtenção do título de mestrado não seriam possíveis. 


\section{Resumo}

Wissmann, Jorge Augusto.; Andrade, Sebastião Arthur Lopez. Estudo de Vigas de Aço com Aberturas de Alma Tipo Alveolar, Litzka e Vigas Expandidas em Altura. Rio de Janeiro, 2009. 136p. Dissertação de Mestrado - Departamento de Engenharia Civil, Pontifícia Universidade Católica do Rio de Janeiro.

Estruturas cada vez mais esbeltas têm sido usadas para otimizar edifícios de grande porte e reduzir custos, inclui-se nisto o pé-direito das edificações e portanto a altura das vigas. Grandes vãos são cada vez mais comuns,

principalmente quando se utiliza aço em edificações, gerando então amplos espaços de serviço em uma direção, mas obstáculos na outra pela continuidade das vigas. A utilização de aberturas na alma das vigas para passagem de tubulações de ar-condicionado, incêndio, etc., evita um acréscimo na altura. Em consequência há uma grande diminuição da capacidade resistente nestes elementos estruturais que dependem diretamente da forma, do tamanho e da posição destas aberturas. A presente dissertação descreve alguns métodos construtivos de aberturas em almas de vigas de aço, cálculos de dimensionamentos e normas relacionadas. Alguns estudos numéricos com aplicações do método dos elementos finitos foram realizados investigando: angulação de aberturas hexagonais, vigas com três aberturas nos terços médios, vigas casteladas (tipo Litzka, com quantidades diferentes de aberturas), vigas expandidas em altura e uma viga Litzka Expandida. Para tal, utilizou-se de seis perfis de uso prático e comparando-os entre estas alternativas descritas. A viga Litzka Expandida se destacou em resistência quando comparada com outros métodos de fabricação, como também pelo menor peso linear, praticidade na fabricação e posicionamento das aberturas.

\section{Palavras-chave}

Análise pelo método dos elementos finitos; Mecanismo de Vierendeel; Abertura na alma; Curvas de interação flexão-cisalhamento; Não linearidade física e geométrica; Vigas de aço, Vigas casteladas, Vigas alveolares, Vigas Litzka, Vigas Expandidas, Viga Litzka Expandida, Análise paramétrica. 


\section{Abstract}

Wissmann, Jorge Augusto.; Andrade, Sebastião Arthur Lopez (advisor). Study of Steel Beams with Web Openings Including Cellular, Litzka and Depth-Expanded Beams. Rio de Janeiro, 2009. 136p. MSc. Dissertation - Departamento de Engenharia Civil, Pontifícia Universidade Católica do Rio de Janeiro.

More slender structures are used in order to reduce costs, floors depht and floor to ceiling clear heights. Steel construction and composite construction for buildings often employs long spans for beams and girders, offering service room between beams in one direction only. The use of web openings for passage of service ducts avoids increasing the depth of construction. Beams with web openings have reductions on flexural and shear resistances depending on the geometry, size and position of such openings on the beam. This dissertation presents some construction methods of beam web openings, analysis and design recommendations, related standards and codes. Some numerical studies by the finite elements method were carried out and results are presented for: type of openings, number and spacing of openings, castelled beams (Litzka type and nonuniform distribution of openings) and expanded in depth beams. For this purpose, six commonly used profiles were used for testing the described web openings solutions. Some comparison results are presented. The Litzka type of web expanded beam presented the best performance in terms of strength, easy of fabrication and reduction of material costs.

\section{Keywords}

Finite Element Analysis; Vierendeel Mechanism; Web Opening; Momentshear Interaction Curves; Material and Geometrical Non-linearity; Steel Beams, Castellated Beams, Cellular Beams, Litzka Beams, Depth-expanded Beams, Litzka Depth-expanded Beams, Parametric Analysis. 


\section{Sumário}

1 Introdução 20

1.1. Motivos e Utilizações 20

1.2. Objetivo 21

1.3. Organização do Trabalho 22

2 Revisão Bibliográfica 23

2.1. Aberturas em Vigas de Aço 23

2.1.1. Formas e Variações 25

2.1.1.1. Tipos 25

2.1.1.2. Aberturas Alongadas 26

2.1.1.3. Excentricidade 27

2.1.2. Viga Castelada $\quad 27$

2.1.2.1. Viga Litzka (Peiner) 28

2.1.2.2. Viga Celular 30

2.1.2.2.1. Vigas de Inércia Variável 31

2.1.2.3. Viga Angelina 32

2.1.3. Viga Expandida 33

2.1.4. Comparações entre aberturas 34

3 Análise dos Esforços 36

3.1. Esforços no Entorno da Abertura 36

3.2. Modos de Falhas 45

3.2.1. Flexão 46

3.2.2. Cisalhamento 46

3.2.3. Mecanismo de Vierendeel 46

3.2.4. Flambagem Local da Mesa Comprimida 47

3.2.5. Flambagem Local da Alma 48

$\begin{array}{ll}\text { 3.3. Reforços Estruturais } & 48\end{array}$ 
3.4. Procedimentos de Dimensionamento 50

3.4.1. Aberturas sem Reforço 50

3.4.1.1. Excentricidade 63

3.4.2. Aberturas Reforçadas 67

3.5. Normas 70

4 Modelo Numérico Computacional 73

4.1. Introdução 73

4.2. Elemento Finito 73

4.3. Malha 74

4.4. Hipóteses Simplificadoras 76

4.5. Software Ansys 76

4.6. Análise Paramétrica $\quad 77$

4.7. Geometria dos Modelos de Validação 77

4.8. Validação do Modelo

5 Estudo Computacional 82

5.1. Abordagem Local de Redwood 82

5.2. Estudo Paramétrico 84

5.2.1. Angulação de Abertura Hexagonal Isolada 86

5.2.2. Estudo de Perfis 94

5.2.2.1. Vigas Originais 96

5.2.2.2. Viga Original com Três Aberturas 97

5.2.2.3. Viga Expandida com Três Aberturas 104

$\begin{array}{ll}\text { 5.2.2.4. Viga Litzka } & 107\end{array}$

6 Viga Litzka Expandida 109

6.1.1. Litzka Expandida Alongada 112

6.1.2. Padrão de Comparação Litzka Expandida 116

6.2. Comparações com Resultados Anteriores 117

6.2.1. Variações para o perfil W200 118

6.2.2. Variações para o perfil W250 119

6.2.3. Variações para o perfil W310 120

$\begin{array}{ll}\text { 6.2.4. Variações para o perfil W360 } & 121\end{array}$ 
6.2.5. Variações para o perfil W410 122

6.2.6. Variações para o perfil W460 123

7 Considerações Finais 125

7.1. Introdução 125

7.2. Resultados Alcançados 125

7.3. Sugestões para Trabalhos Futuros 127

8 Referências Bibliográficas 128

Anexo A - Curvas de Interação Flexão-cisalhamento 132

$\begin{array}{ll}\text { Anexo B - Exemplos de Dimensionamento } & 137\end{array}$ 


\section{Listas de Figuras}

Figura 1.1- Representação da flexibilidade na fabricação de viga castelada $\quad 20$

Figura 1.2 - Passagens de tubulações em aberturas de vigas de aço [24] 21

Figura 2.1 - Centro de Convenções World Trade Center, São Paulo [18] 23

Figura 2.2 - Pórtico de Catálogo da Gerdau Açominas [12] 24

Figura 2.3 - Configurações geométricas mais comuns de aberturas na alma 25

Figura 2.4 - Abertura alongada e sua flexibilidade [5] 26

Figura 2.5 - Abertura circular alongada aparelhada para ensaio [19] 26

Figura 2.6 - Representação de viga celular com excentricidade 27

Figura 2.7 - Corte na alma em padrão trapezoidal 27

Figura 2.8 - Resultado final do transpasse e solda 28

Figura 2.9 Ensaio de viga castelada [21] 28

Figura 2.10 - Ilustração do dimensionamento de uma viga Litzka [11] 29

Figura 2.11 - Fabricação de viga celular (duas linhas de corte) 30

Figura 2.12 - Fabricação automatizada da viga celular [19] 31

Figura 2.13 - Transpasse, solda e sobra (scrap) 31

Figura 2.14 - Tapered ACB Beam [19] 32

Figura 2.15 - Viga Angelina [2] 32

Figura 2.16 - Corte da viga original e da chapa de expansão 33

Figura 2.17 - Resultado da viga expandida 33

Figura 2.18 - Viga Castelada expandida 34

Figura 2.19 - Comparativo de seções de aberturas [1] 35

Figura 3.1 - Ilustração dos "tês" e das variáveis utilizadas por Redwood [15] 36

Figura 3.2 - Localização das rótulas plásticas Redwood [15] 38

Figura 3.3 - Distribuição de forças na seção perfurada [5] 39

Figura 3.4 - Mecanismo de Vierendeel no entorno de uma abertura circular, Chung et at [6] 40

Figura 3.5 - Exemplo de curvas de interação flexão-cisalhamento obtidas por investigações em elementos finitos [5] 42

Figura 3.6 - Formas e variáveis das aberturas estudadas por Chung et al [5] 43 
Figura 3.7 - Redução na curva de iteração flexão-cisalhamento de uma seção perfurada devido ao mecanismo de Vierendeel [5] 47

Figura 3.8 - Tipos de Reforço para Aberturas na Alma - Redwood [15] 49

Figura 3.9 - Região adicional considerada em um "T", dada pela mesa da viga [5]

Figura 3.10 - Curvas de iteração flexão-cisalhamento propostas [5] 56

Figura 3.11 - Valores típicos do parâmetro Vierendeel para um perfil UB 457x152x52 S275 [5] 57

Figura $3.12-v_{i}$, v e m das seções perfuradas ao longo do vão da viga. Perfil UB $457 x 152 x 52$ S275 [5] 59

Figura 3.13 - Análise dos esforços assumindo plastificação, Redwood [15] 60

Figura 3.14 - Diagrama de interações momento-cortante para o perfil IPE550 63

Figura 3.15 - Abertura circular excêntrica sujeita a momento e cortante, Redwood [15]

Figura 3.16 - Detalhes e notações da abertura na alma utilizadas por Redwood e $\begin{array}{ll}\text { Shrivastava [17] } & 68\end{array}$

Figura 3.17 - Ilustração da “zona neutra” segundo Veríssimo [24] 71

Figura 3.18 - Condições para execução de aberturas circulares [3] 72

Figura 4.1 - Elemento SHELL 281, 8-Node Finite Strain Shell [10] 74

Figura 4.2 - Seqüência de modelagem representando linhas, áreas, malha de $\begin{array}{ll}\text { elementos carregamento } & 75\end{array}$

Figura 4.3 - Modelo elaborado para validação do estudo 75

Figura 4.4 - Detalhes da modelagem utilizada por Chung et al [6] 78

Figura 4.5 - Localização da abertura e da aplicação da carga, Viga3A 78

Figura 4.6 - Diagrama de momento fletor e representação de esforços em seções da Viga3A $\quad 79$

Figura 4.7 - Solução Nodal de Tensões pelo critério de Von Mises, Viga3A 79

Figura 4.8 - Comparação da formação de rótulas plásticas conforme literatura 80

Figura 4.9 - Resultados obtidos por Chung [6] para vigas 2A e 3A 80

Figura 4.10 - Comparação com os ensaios Chung [6], obtidos através dos modelos criados para VIGA2A e 3A $\quad 81$

Figura 5.1 - Curva de interação M-V para viga IPE550 com abertura 50\% da altura da viga 
Figura 5.2 - Curva de interação M-V para viga ipe550 com abertura 70\% da altura da viga

Figura 5.3 - Variáveis utilizadas na presente pesquisa, forma hexagonal alongada

Figura 5.4 - Esboço dos esforços de momento fletor e esforço cortante respectivamente, nas aberturas da VIGA3A

Figura 5.5 - Estudo paramétrico no ângulo de abertura hexagonal 2:1 isolada para o braço de $762 \mathrm{~mm}$

Figura 5.6 - Distribuição de tensões Von Mises para os ângulos simulados das aberturas centradas a $762 \mathrm{~mm}$ do apoio esquerdo, com o comprimento total fixo

Figura 5.7 - Estudo paramétrico no ângulo de abertura hexagonal 2:1 isolada para o braço de $556 \mathrm{~mm}$

Figura 5.8 - Rótulas plásticas desenvolvidas para cada ângulo simulado, a $556 \mathrm{~mm}$ do apoio e com o comprimento total fixo

Figura 5.9 - Estudo paramétrico no ângulo de abertura hexagonal 2:1 isolada para o braço de $350 \mathrm{~mm}$

Figura 5.10 - Nova configuração com o comprimento total da abertura

Figura 5.11 - Estudo paramétrico no ângulo de abertura hexagonal 2:1 isolada para o braço de $762 \mathrm{~mm}$ com 2a fixo

Figura 5.12 - Estudo paramétrico no ângulo de abertura hexagonal 2:1 isolada para o braço de $556 \mathrm{~mm}$ com 2a fixo

Figura 5.13 - Estudo paramétrico no ângulo de abertura hexagonal 2:1 isolada para o braço de $350 \mathrm{~mm}$ com 2a fixo

Figura 5.14 - Rótulas plásticas desenvolvidas para cada ângulo simulado, a 350 mm do apoio e com o comprimento total variável

Figura 5.15 - Aplicação de deslocamentos nos terços e esforços

Figura 5.16 - Curvas de resistência dos perfis originais, sem aberturas

Figura 5.17 - Modelo da viga W200 com três aberturas, representação das áreas 98

Figura 5.18 - Curvas de resistência dos perfis originais com três aberturas para a tabela de configuração 1

Figura 5.19 - Viga W200 com três aberturas, tensão Von Mises em MPa, configuração 1 
Figura 5.20 - Viga W200 com três aberturas, deformação plástica Von Mises, configuração 1

Figura 5.21 - Viga W460 com três aberturas, Von Mises Strain-Plastic, configuração 1

Figura 5.22 - Curvas de resistência dos perfis originais com três aberturas para a tabela de configuração 2

Figura 5.23 - Formação de rótulas plásticas para a viga W200 com três aberturas para a configuração 2

Figura 5.24 - Formação de rótulas plásticas para a viga W460 com três aberturas, configuração 2

Figura 5.25 - Representação da chapa de expansão em vermelho com outra espessura

Figura 5.26 - Simulação da viga W200 + chapa de 100 mm, com três aberturas, Von Mises Stress

Figura 5.27 - Curvas de resistência dos perfis com chapa de expansão e três aberturas para a configuração 1

Figura 5.28 - Curvas de resistência dos perfis mais chapa de expansão com três aberturas para a configuração 2

Figura 5.29 - Plastificação no lado de menor momento para perfil W250 para configuração 2

Figura 5.30 - Modelo de viga Litzka W200 (áreas), com 27 aberturas 107

Figura 5.31 - Curvas de resistência dos perfis em forma de viga Litzka 108

Figura 6.1 - Modelo de Viga Litzka Expandida demonstrando espessuras das chapas

Figura 6.2 Litzka expandida W200, tensões para configuração 1

Figura 6.3 Litzka expandida W200, tensões para configuração 2

Figura 6.4 Litzka expandida W250, tensões para configuração 3

Figura 6.5 Litzka expandida W360, tensões para configuração 4

Figura 6.6 - Curvas de resistência dos perfis Litzka Expandida

Figura 6.7 - Litzka Expandida W200 com quatro aberturas alongadas, configuração 5

Figura 6.8 Litzka expandida W200, deformações plásticas para configuração 5113 Figura 6.9 - Litzka Expandida W200 com quatro aberturas alongadas, configuração 6 
Figura 6.10 Litzka Expandida W200, deformações plásticas para configuração 6

Figura 6.11 - Configurações simuladas da viga Litzka Expandida W200 114 Figura 6.12 Litzka expandida W410, deformações plásticas para configuração 7

Figura 6.13 Litzka expandida W460, deformações plásticas para configuração 8

Figura 6.14 - Resistências das vigas Litzka Expandidas com aberturas alongadas

116

Figura 6.15 - Curvas de resistência dos perfis com dimensões de Litzka Expandida mas com alma cheia

Figura 6.16 - Curvas de resistência para as simulações do perfil W200

Figura 6.17 - Curvas de resistência para as simulações do perfil W250 119

Figura 6.18 - Curvas de resistência para as simulações do perfil W310 120

Figura 6.19 - Curvas de resistência para as simulações do perfil W360 121

Figura 6.20 - Curvas de resistência para as simulações do perfil W410 122

Figura 6.21 - Curvas de resistência para as simulações do perfil W460 123 


\section{Listas de Tabelas}

Tabela 3.1 - Aumento da resistência ao cisalhamento fornecido pela nova consideração da mesa [5]

Tabela 3.2 - Algumas relações de resistência dupla, $\bar{v}$, para diferentes relações

$$
\frac{d_{0}}{h} \text {, examinadas por Chung et al [5] }
$$

Tabela 4.1 - Comparativo de resultados com Chung para Vigas 2A e 3A

81

Tabela 5.1 - Novos comprimentos totais das aberturas

91

Tabela 5.2 - Perfis estudados

94

Tabela 5.3 - Configurações das Vigas Litzka

95

Tabela 5.4 - Configuração um, para vigas com três aberturas

98

Tabela 5.5 - Configuração dois, para vigas com três aberturas

101

Tabela 6.1 - Peso dos perfis estudados com alma cheia mas com altura Litzka Expandida

Tabela B.1 - Esforços solicitantes em cada abertura do exemplo B.1

138

Tabela B.2 - Esforços solicitantes em cada abertura do exemplo B.2 


\section{Lista de Símbolos}

$E$ é o módulo de elasticidade do aço;

$f_{y}$ é a resistência ao escoamento do aço;

$\alpha$ é o ângulo formado pela abertura hexagonal;

$2 \mathrm{H}$ é a altura vertical da abertura;

2a é o comprimento da abertura ;

d é a altura total do perfil;

b é largura da mesa;

w é a espessura da alma;

t é a espessura da mesa;

L é o comprimento da viga ;

echapa é a espessura da chapa de reforço;

hchapa é a altura da chapa de expansão;

Hfinal é a altura final do perfil modificado;

$\mathrm{N}$ é o número de aberturas (para vigas Litzka).
Abreviaturas
ACB - ArcelorMital Cellular Beam
AISC - American Institute of Steel Construction
APDL - Ansys Parametric Design Language 
CBCA - Centro Brasileiro da Construção em Aço

CSA - Canadian Standards Association

EC3- Eurocode 3

ECCS - European Convention for Constructional Steelwork

EN - European standard

ENV - European pre-standard

ENV - Eurocode - Version of Eurocode published by CEN as a pre-standard

ENV - (for subsequent conversion into EN)

HMS - High Moment Side (lado de maior momento)

LMS - Low Moment Side (lado de menor momento)

MEF - Método dos Elementos Finitos

UB - Universal Beam 
Toda a nossa ciência, comparada com a realidade, é primitiva e infantil e, no entanto, é a coisa mais preciosa que temos.

Albert Einstein 